\title{
Spin-charge separation in Aharonov-Bohm rings of interacting electrons
}

\author{
K. Hallberg, ${ }^{1}$ A. A. Aligia, ${ }^{1}$ A. P. Kampf, ${ }^{2}$ and B. Normand ${ }^{3}$ \\ ${ }^{1}$ Centro Atómico Bariloche and Instituto Balseiro, \\ Comisión Nacional de Energía Atómica, 8400 Bariloche, Argentina \\ ${ }^{2}$ Institut für Physik, Theoretische Physik III, Elektronische Korrelationen und Magnetismus, \\ Universität Augsburg, 86135 Augsburg, Germany \\ ${ }^{3}$ Département de Physique, Université de Fribourg, CH-1700 Fribourg, Switzerland
}

\begin{abstract}
We investigate the properties of strongly correlated electronic models on a flux-threaded ring connected to semi-infinite free-electron leads. The interference pattern of such an Aharonov-Bohm ring shows sharp dips at certain flux values, determined by the filling, which are a consequence of spin-charge separation in a nanoscopic system.

PACS numbers: 75.40.Gb, 75.10.Jm, 76.60.Es
\end{abstract}

In interacting electron systems in reduced spatial dimensions, correlation effects are strongly enhanced and the conventional quasiparticle description of Fermi liquids may become inapplicable. In particular, for onedimensional (1d) systems the low-energy excitations are entirely collective in nature and the Luttinger-liquid (LL) concept provides the appropriate framework to characterize the electronic properties. A hallmark of the LL is the fractionalization of the electronic excitations into separate collective spin and charge modes, a phenomenon known as spin-charge separation (SCS) [1, 2].

With the advent of new materials and artificial structures of quasi- $1 \mathrm{~d}$ electronic character in the last decade, a variety of experiments has been reported which seek evidence of SCS. Prominent examples of candidate materials include the organic Bechgaard and Fabre salts [3], molybdenum bronzes and chalcogenides [4], cuprate chain and ladder compounds [5] and also carbon nanotube systems [6]. The non-Fermi-liquid normal-state properties of high temperature superconductors have also led to attempts to trace their origin to the possible realization of SCS in strongly correlated electron systems in $2 \mathrm{~d}$ [7]. Different approaches for the identification of SCS have included the analysis of non-universal power-law $I$ - $V$ characteristics [4], the search for characteristic dispersive features by angle-resolved photoemission spectroscopy (ARPES) 8], establishing a violation of the Wiedemann-Franz law [9], and analyzing spin and charge conductivities [8, 10]. While the interpretation of experimental results has been considered ambiguous in some cases, a verification of SCS has been reported from ARPES data on $\mathrm{SrCuO}_{2}$ [1].

Theoretical methods for detecting and visualizing SCS were proposed and demonstrated many years ago. Direct calculations of the real-time evolution of electronic wave packets in Hubbard rings revealed that the spin and charge densities dispersed with different velocities as an immediate consequence of SCS 12. Equally striking was the analysis of transmission through AharonovBohm (AB) rings [13]. The motion of the electrons in the ring was described by a LL propagator, where different charge and spin velocities, respectively $v_{c}$ and $v_{s}$, are included explicitly. With this assumption the fluxdependence of the transmission is no longer periodic only in multiples of a flux quantum $\Phi_{0}=h c / e$, but instead new structures appear at fractional flux values which are determined by the ratio $v_{s} / v_{c}$. In essence, these structures arise because transmission requires the separated spin and charge degrees of freedom of an injected electron to recombine at the drain lead after traveling through the ring in the presence of the $\mathrm{AB}$ flux.

In this letter we propose an experimental configuration which may serve as a clean and direct probe of SCS. We employ the AB-ring transmission, focusing primarily on the $t$ - $J$ model as a prototypical interacting system relevant to artificially designed $1 \mathrm{~d}$ nanostructures such as small rings of quantum dots. We find a clear reduction of the transmittance of such a device at magnetic fields corresponding to fractional values of the flux in units of the flux quantum. These are explained in terms of an analysis of the momentum quantum numbers of the spin and charge excitations, and of the orbital-flux-induced phase shifts accumulated on traversing the ring between the two contact leads. The flux-dependent interference pattern in the transmission through an $\mathrm{AB}$ ring is thus shown to be a valid and feasible tool for the unambiguous detection of certain signatures of the SCS phenomenon.

The system in Fig. 1(a) has the Hamiltonian

$$
H=H_{\text {leads }}+H_{\text {link }}+H_{\text {ring }},
$$

where

$$
H_{\text {leads }}=-t \sum_{i=-\infty, \sigma}^{-1} a_{i-1, \sigma}^{\dagger} a_{i, \sigma}-t \sum_{i=1, \sigma}^{\infty} a_{i, \sigma}^{\dagger} a_{i+1, \sigma}+\text { H.c. }
$$

describes free electrons in the left and right leads,

$$
H_{\text {link }}=-t^{\prime} \sum_{\sigma}\left(a_{-1, \sigma}^{\dagger} c_{0, \sigma}+a_{1, \sigma}^{\dagger} c_{L / 2, \sigma}+\text { H.c. }\right)
$$

describes the exchange of quasiparticles between leads 


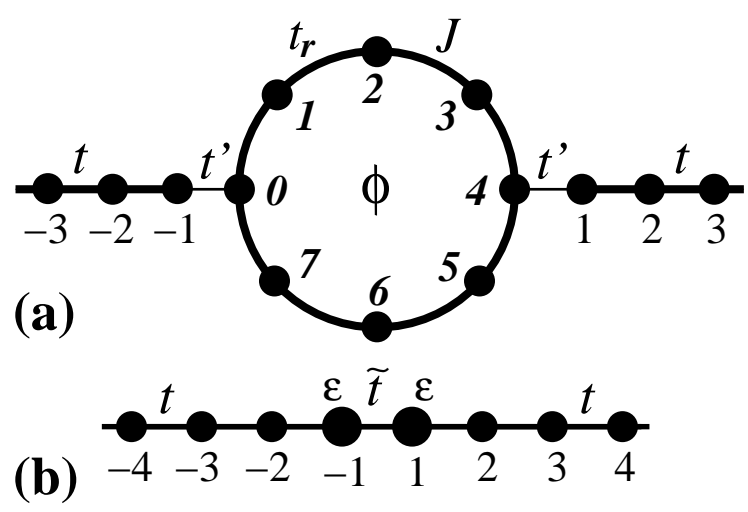

FIG. 1: (a) Schematic representation of an interacting system on a ring connected by links $t^{\prime}$ to free-electron leads. The number of sites in the ring depicted is $L=8$, and the transmittance is computed from the Green function connecting sites 0 and $L / 2$. (b) Effective model of two impurities in a single conducting chain derived in the limit of weak $t^{\prime}$.

$\left(a_{i, \sigma}\right)$ and ring $\left(c_{l, \sigma}\right)$, and

$$
\begin{aligned}
H_{\text {ring }}= & -e V_{g} \sum_{l, \sigma} c_{l, \sigma}^{\dagger} c_{l, \sigma}-t_{r} \sum_{l, \sigma}\left(c_{l, \sigma}^{\dagger} c_{l+1, \sigma} e^{-i \phi / L}\right. \\
& + \text { H.c. })+H_{\mathrm{int}}
\end{aligned}
$$

describes the interacting electron system. The $\mathrm{AB}$ ring has length $L$, is threaded by flux $\phi$, where $\phi=2 \pi \Phi / \Phi_{0}$, and is subject to an applied gate voltage $V_{g}$.

Following Ref. 13], the transmission from the left to the right lead can be calculated to second order in $t^{\prime}$ from an effective low-energy Hamiltonian $H_{\text {eff }}$ for the system with an additional particle of energy $\omega$ and wave vector $\pm k$ in the left or the right lead. $H_{\text {eff }}$ is equivalent to the one-particle Hamiltonian for the chain represented in Fig. 1(b), with effective energy $\epsilon(\omega)=t^{\prime 2} G_{0,0}^{\mathrm{R}}(\omega)$ for sites adjacent to the ring [ -1 and 1 in Fig. 1(a)], and effective hopping $\tilde{t}(\omega)=t^{\prime 2} G_{0, L / 2}^{\mathrm{R}}(\omega)$ across the ring; $G_{i, j}^{\mathrm{R}}(\omega)$ denotes the Green function of the isolated ring.

At zero temperature, the transmittance and conductance of the system may then be computed using the effective impurity problem. This proceeds in the $T$-matrix formulation, which yields transmission amplitudes based on an intersite promotion matrix [14, 15]. The transmittance $T(\omega)$ is given by [13]

$$
T\left(\omega, V_{g}, \phi\right)=\frac{4 t^{2} \sin ^{2} k|\tilde{t}(\omega)|^{2}}{\left.\left|\left[\omega-\epsilon(\omega)+t e^{i k}\right]^{2}-\right| \tilde{t}^{2}(\omega)\right|^{2}},
$$

where $\omega=-2 t \cos k$ is the tight-binding dispersion relation for the free electrons in the leads which are incident upon the impurities. These equations are exact for a non-interacting system, while with interactions on the ring they serve as an approximation in the tunneling limit $t^{\prime} / t \ll 1[13]$. We comment that previous calculations of $T\left(\omega, V_{g}\right)$ in nanoscopic systems do not include

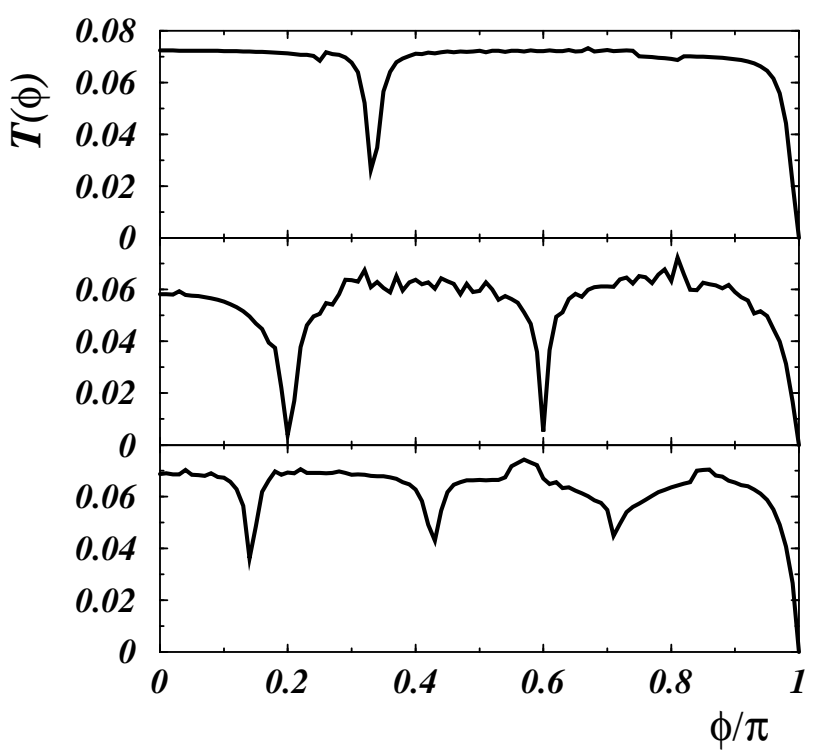

FIG. 2: Transmittance as a function of flux for a $t_{r^{-}} J$ model with $J=0.001 t_{r}, t_{r}=t, t^{\prime}=0.3 t$, and $L=8$ sites. The filling of the ring is (a) $N+1=4$, (b) $N+1=6$, and (c) $N+1=8$. The transmission occurs through intermediate states with $N=3,5$, and 7 particles, respectively, which lead to minima at flux values $\phi_{d} \approx \pi\left(1-2 n_{s} / N\right)$ (see text).

interference effects which exist in a ring geometry. We emphasize that this analysis is applicable only for systems in which the ground state before and after particle or hole injection has no spin degeneracy. Thus Eq. (5) does not include the Kondo effect, which arises in the spin-degenerate case, but in any event is destroyed even by small temperatures or applied magnetic fields.

To calculate $T\left(\omega, V_{g}\right)$ we employ numerical diagonalization of the isolated ring using a $t_{r^{-}} J$ model where $H_{\mathrm{int}}=J \sum_{l}\left(\mathbf{S}_{l} \cdot \mathbf{S}_{l+1}-1 / 4\right)$ in Eq. (4), with $\mathbf{S}_{l}=$ $\sum_{\alpha \beta} c_{l \alpha}^{\dagger} \sigma_{\alpha \beta} c_{l \beta}$ the spin at site $l$ and implicit projection of the $t_{r}$ term to single site occupancy. We consider particles incident on a ring of $L$ sites and $N+1$ electrons, obtaining the Green functions from the ground state of the isolated ring [16] and substituting these in Eq. (5). We fix the energy $\omega=0$ to represent half-filled leads and explore the dependence of the transmittance on the flux. $T\left(0, V_{g}\right)$ as a function of $V_{g}$ shows narrow peaks (with a width proportional to $\left.\left(t^{\prime}\right)^{2}\right)$ at gate voltages which match the excitation energies of the system.

The transmittance of the ring, obtained by integration over the excitations in a small energy window at the Fermi level [13], is shown in Fig. 2] for different ring fillings. For injection of a hole in systems containing $N+1=4,6$ or 8 particles [Fig. 2(a)-(c)], the dynamical properties correspond to $N=3,5$, or 7 particles. The most striking result is the existence of dips at certain flux values, which constitute a clear signature of SCS.

A first obvious feature is that the transmittance van- 
ishes at flux $\phi=\pi$. This is expected from negative interference of the components of the electron wave function traveling in the upper and lower halves of the ring. Formally, it is a consequence of the reflection symmetry of the device through the axis containing the leads. For $\phi=\pi$, the gauge transformation

$$
f_{l, \sigma}^{\dagger}=c_{l, \sigma}^{\dagger} e^{i l \phi / L}, g_{m, \sigma}^{\dagger}=a_{m, \sigma}^{\dagger} e^{i \phi / 2}, m>0
$$

leads to a Hamiltonian with all hoppings real and of the same sign, except between ring sites 0 and $L-1$, where it has the opposite sign. The transformed Hamiltonian is clearly invariant under simultaneous reflection and sign change of the phase of $c_{0, \sigma}^{\dagger}\left(c_{l, \sigma}^{\dagger} \leftrightarrow c_{L-l, \sigma}^{\dagger}\right.$ for $l>0$, $c_{0, \sigma}^{\dagger} \leftrightarrow-c_{0, \sigma}^{\dagger}$ for $l=0$ ), whereas the Green function $G_{0, L / 2}^{\mathrm{R}}(\omega)$ changes sign. The latter must be invariant with respect to operators of the symmetry group of $H$, and therefore vanishes along with $T(\omega)$ (5) .

While the presence of the dip at $\phi=\pi$ is quite general, the origin of the other dips in the transmittance resides in the strongly correlated nature of the problem. We find numerically that the peaks are better defined for small interaction strengths $\left(J / t_{r}<1 / L\right)$, as discussed below, and therefore begin our explanation of the presence of the additional dips by assuming $J=0$. In this limit the model is equivalent to the infinite- $U$ Hubbard model, and complete SCS takes place on all energy scales [17, 18].

Following the method of Ref. [19] for the ring with arbitrary flux, we construct spin wave functions which transform under the irreducible representations of the group $C_{N}$ of cyclic permutations of the $N$ spins of the $L$-site system. Each of these representations is labeled by a wave vector $k_{s}=2 \pi n_{s} / N$, where the integer $n_{s}$ characterizes the spin wave function. For $J=0$ each element of $C_{N}$ commutes with $H_{\text {ring. In each subspace of states }}$ whose spin wave function is characterized by the quantum number $k_{s}$, the problem may be mapped to that of a non-interacting, spinless system with effective flux [19]

$$
\phi_{\mathrm{eff}}=\phi+k_{s}=\phi+2 \pi n_{s} / N
$$

The total energy of any state of the ring becomes

$$
E=-2 t_{r} \sum_{l=1}^{N} \cos \left(k_{l}+\frac{\phi_{\mathrm{eff}}}{L}\right), \quad k_{l}=\frac{2 \pi}{L} n_{l}
$$

where $n_{l}$ and $N$ are charge quantum numbers. Thus the dynamical charge properties are described completely by a spinless model. The SCS phenomenon enters in that the spin wave function modifies the effective flux seen by the charges. Hole injection in an $(N+1)$-particle system (or particle injection for an ( $N-1)$-particle system) yields an intermediate $N$-particle state with a certain weight for each spin wave number $k_{s}$, before the hole (particle) is ejected through the other lead. Because the charge

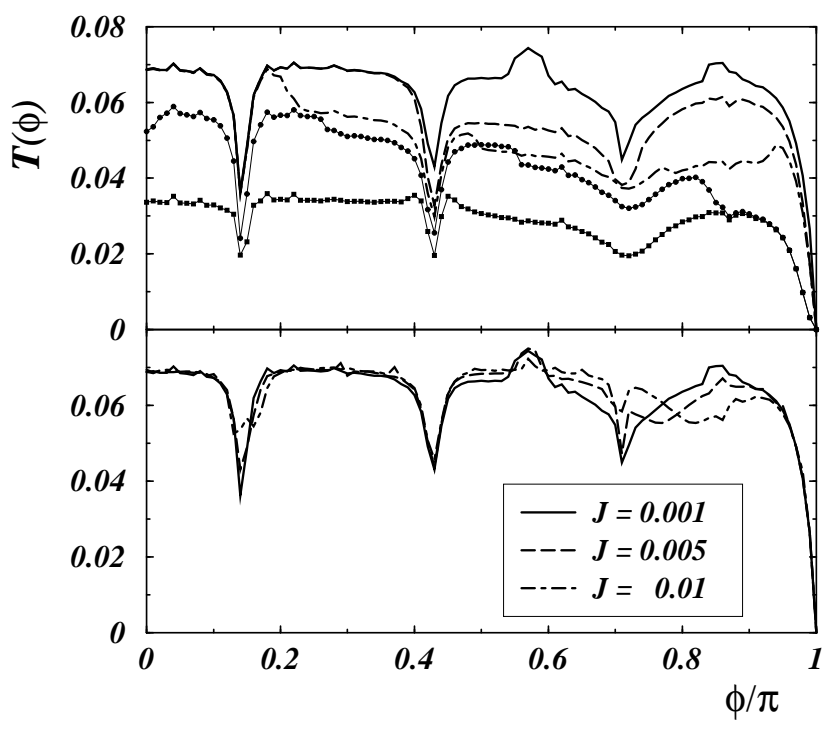

FIG. 3: (a) Transmittance as a function of flux for a system of $L=8$ with $J=0.001 t_{r}, N+1=8$ particles and different window sizes ranging from $0.6 t$ to $0.15 t$ (top to bottom) below the Fermi energy. (b) As (a) for a selection of values of $J$.

dynamics in the ring are determined by the flux $\phi_{\text {eff }}\left(k_{s}\right)$, states with spin wave numbers $k_{s}$ do not contribute to the transport when $\phi_{\text {eff }}\left(k_{s}\right)=\pi$ because they interfere destructively (above). From Eq. (17) one therefore expects dips in the transmittance when $\phi=\phi_{d}$ with

$$
\phi_{d}=\pi\left(1-2 n_{s} / N\right)
$$

In Fig. 2 we have considered the transmission contributions of excitations in a finite energy window, which accounts for possible (gate and bias) voltage fluctuations and temperature effects unavoidably present in an experimental system. For given $\phi_{d}\left(n_{s}\right)$, the depth of the dip in the integrated transmittance depends on the number of energy levels which fall inside the window. If for given $V_{g}$ the window includes the destructively interfering states, then $T(\phi)$ exhibits dips at flux $\phi_{d}$. The dependence of the dip structures on the size of the window is shown in Fig. 3(a) for a system with $N+1=8$ particles. Although the integrated transmission decreases with window width, the principal features remain present, indicating the origin of the dips in destructive interference of levels very close to the Fermi energy. If the window size is too large (not shown), the dip depth diminishes due to the presence of additional, non-interfering peaks further from the Fermi level.

Our numerical results indicate that the above reasoning remains valid for finite $J$, where SCS is incomplete. For $J=0$, the lowest-energy states with consecutive charge quantum numbers [19] include in general all possible $k_{s}$. We find a schematic correspondence of these states to effective "spin" subbands separated by a charge energy scale on the order of the finite-size gap of the ring, 
$t_{r} / L$. SCS is then expected to remain a valid concept for the spin and charge excitations of a small system for $J / t_{r}<1 / L$, a result confirmed by the progressive loss of well-defined dip structures in Fig. 3(b). The breakdown of SCS may then be described as an intrinsic phenomenon related to the mixing of different charge subbands. This mixing is strongest at higher values of the flux [Fig. 3(b)]. For small $J$ the position of the dips is shifted slightly, but remains close to the flux values given by Eq. (9) for all fillings shown in Fig. 2.

We summarize briefly the connection between the destructive interference of certain excited states, which is responsible for the transmittance dips, and the concept of separate, effective spin and charge velocities $v_{s}$ and $v_{c}$ in the ring, as observed by considering the evolution of electronic wave packets [12. In the limit $U \rightarrow \infty, v_{s}$ and $v_{c}$ can be obtained from the ratio of the change in total energy and momentum when either $n_{s}$ or one of the $n_{l}$ is increased by one, using either Eqs. (7) and (8) or the Bethe-Ansatz equations of Ref. 17]. For the $\mathrm{N}$ particle intermediate states relevant to our analysis, the effective spinless model has flux $\pi$ and odd $N$, circumstances under which for spin quantum number $n_{s}$ and flux $\phi_{d}\left(n_{s}\right)$ one obtains $v_{s} / v_{c}=1 / N$. This expression is then consistent with a qualitative understanding of the dips in Figs. 2(a)-(c) in terms of the charge and spin components of the injected particle performing different numbers of turns around the ring before reuniting at the drain lead. We stress, however, that in small $t_{r^{-}} J$ and Hubbard rings a distribution in effective velocities for the states involved in transmission processes is unavoidable at arbitrary flux values.

Transmittance dips should also be observable in an Aharonov-Casher (AC) geometry, where the ring is pierced by a charged wire 20,21], by extending the treatment to the case in which different fluxes act on particles with up- and down-spins 22]. Specifically, the mapping of charge degrees of freedom to a spinless model for $J=0$ remains applicable with the substitution $\Phi \rightarrow \Phi+\sigma_{z} \Phi_{\mathrm{AC}}$ in Eq. (7), where $\sigma_{z}$ is the spin projection on the axis of the ring and $\Phi_{\mathrm{AC}}$ is proportional to the radial electric field 21. An experimental realization of the $\mathrm{AB}$ system requires the design of artificial structures, such as rings of quantum dots, on the sub- $\mu \mathrm{m}$ scale; accessible laboratory fields will not permit $\mathrm{AB}$ experiments on molecular rings. The wide variety of quantum-dot assemblies synthesized in recent years 23] suggests that such structures are well within the compass of current nanofabrication technology [24]. Similar devices with a charged central gate could be used for measurements of the AC effect, which may also be feasible on a more molecular scale with multi-wall nanotubes, charged nanotubes piercing large molecular rings, or closed-loop nanotube assemblies.

In conclusion, the transmittance through $\mathrm{AB}$ rings of interacting electrons provides a straightforward technique for the detection of SCS. The existence of transmis- sion dips arising from non-trivial destructive interference effects at fractional values of the flux quantum $\Phi_{0}$ is a robust signature of SCS. While the depths and widths of the dips vary with the system under consideration, their positions depend only on ring filling and weakly on interaction strengths. The experimental capability to construct systems exhibiting nanoscopic SCS exists already.

We are grateful to G. Japaridze and M. Sekania for helpful discussions. K.H. and A.A.A. are fellows of CONICET, and thank the Fundación Antorchas, Project 14116-168, and PICT 03-12742 of ANPCyT for support. A.P.K. acknowledges the support of the Deutsche Forschungsgemeinschaft through SFB 484 and B.N. the support of the Swiss National Science Foundation.

[1] F. D. M. Haldane, J. Phys. C 14, 2585 (1981).

[2] H. J. Schulz, Int. J. Mod. Phys. B 5, 57 (1991).

[3] C. Bourbonnais and D. Jerome, in Advances in Synthetic Metals, Twenty years of Progress in Science and Technology, Eds. P. Bernier, S. Lefrant, and G. Bidan (Elsevier, New York, 1999).

[4] J. Voit, Rep. Prog. Phys. 58, 977 (1995).

[5] E. Dagotto and T. M. Rice, Science 271, 618 (1996).

[6] R. Egger and A. O. Gogolin, Phys. Rev. Lett. 79, 5082 (1997).

[7] P. W. Anderson, The Theory of Superconductivity in the High- $\mathrm{T}_{c}$ Cuprates, (Princeton University Press, Princeton, 1997).

[8] J. Voit, in Proceedings of the $9^{t h}$ International Conference on Recent Progress in Many-Body Physics, Ed. D. Neilson (World Scientific, Singapore, 1998).

[9] C. L. Kane and M. P. A. Fisher, Phys. Rev. Lett. 76, 3192 (1996); R. W. Hill et al., Nature 414, 711 (2001).

[10] Q. Si, Phys. Rev. Lett. 78, 1767 (1997); Physica C 341, 1519 (2000).

[11] C. Kim et al., Phys. Rev. Lett. 77, 4054 (1996).

[12] E. A. Jagla, K. Hallberg, and C. A. Balseiro, Phys. Rev. B 47, 5849 (1993).

[13] E. A. Jagla and C. A. Balseiro, Phys. Rev. Lett. 70, 639 (1993).

[14] A. D. Stone et al., Phys. Rev. B 24, 5583 (1981).

[15] P. W. Anderson et al., Phys. Rev. B 22, 3519 (1980).

[16] E. Gagliano and C. Balseiro, Phys. Rev. Lett. 59, 2999 (1987).

[17] M. Ogata and H. Shiba, Phys. Rev. B 41, 2326 (1990);

[18] E. Lieb and F. Wu, Phys. Rev. Lett 20, 1445 (1968).

[19] W. Caspers and P. Ilske, Physica A 157, 1033 (1989); A. Schadschneider, Phys. Rev. B 51, 10386 (1995).

[20] Y. Aharonov and A. Casher, Phys. Rev. Lett. 53, 319 (1984).

[21] A. Balatsky and B. Altshuler, Phys. Rev. Lett. 70, 1678 (1993).

[22] L. Arrachea, A. A. Aligia, and E. Gagliano, Phys. Rev. Lett. 76, 4396 (1996).

[23] L. P. Kouwenhoven et al., Phys. Rev. Lett. 65, 361 (1990); C. P. Collier et al., Science 277, 1978 (1997); G. Medeiros-Ribeiro et al., Phys. Rev. B 59, 1633 (1999).

[24] L. P. Kouwenhoven, Science 268, 1440 (1995); A. P. 
Aliviasatos, Science 271, 933 (1996). 